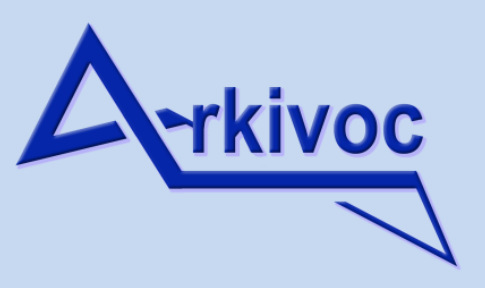

Archive for

Organic Chemistry
The Free Internet Journal

for Organic Chemistry
Paper

Arkivoc 2019, part v, 152-162

\title{
Synthesis and biological evaluation of benzimidazolyl substituted aloe-emodin derivatives
}

\author{
Yennys Hernández-Molina, ${ }^{a, b}$ Guido Verniest, ${ }^{* b}$ Magaly Casals-Hung, ${ }^{a}$ Jorge Acevedo-Martínez, ${ }^{a}$ \\ and Paul $\operatorname{Cos}^{\mathrm{c}}$
}

${ }^{a}$ Department of Chemistry, Faculty of Natural and Exact Sciences, Universidad de Oriente, Patricio Lumumba SN, Santiago de Cuba, 90500, Cuba

${ }^{b}$ Research Group of Organic Chemistry, Department of Chemistry and Department of Bio-Engineering Sciences, Faculty of Science and Bio-Engineering Sciences, Vrije Universiteit Brussel (VUB), Pleinlaan 2, B-1050 Brussels, Belgium

${ }^{c}$ University of Antwerp, Laboratory of Microbiology, Parasitology and Hygiene, Faculty of Pharmaceutical, Biomedical and Veterinary Sciences, Universiteitsplein 1, B-2610 Antwerp, Belgium

Email: gvernies@vub.ac.be

Received 07-08-2018

Accepted 02-05-2019

Published on line 03-13-2019

\section{Abstract}

Benzimidazolic derivatives of the natural product aloe-emodin were prepared in good yields via aerobic condensation of the corresponding aldehydes with diamines in the presence of potassium iodide as auxiliary reagent. The required aldehydes were easily obtained by oxidation of aloe-emodin or its dimethyl-protected analogue. In vitro antimicrobial activity and cytotoxicity of the synthesized compounds were assessed.

Most of the compounds showed either low to moderate antiprotozoal activity, although it was quite aspecific. 3-(1H-Benzo[d]imidazol-2-yl)-1,8-dihydroxyanthracene-9,10-dione showed the highest specificity against Leishmania infantum with $\mathrm{IC}_{50}=4.06 \mu \mathrm{M}$ and a selectivity index of 10.29.

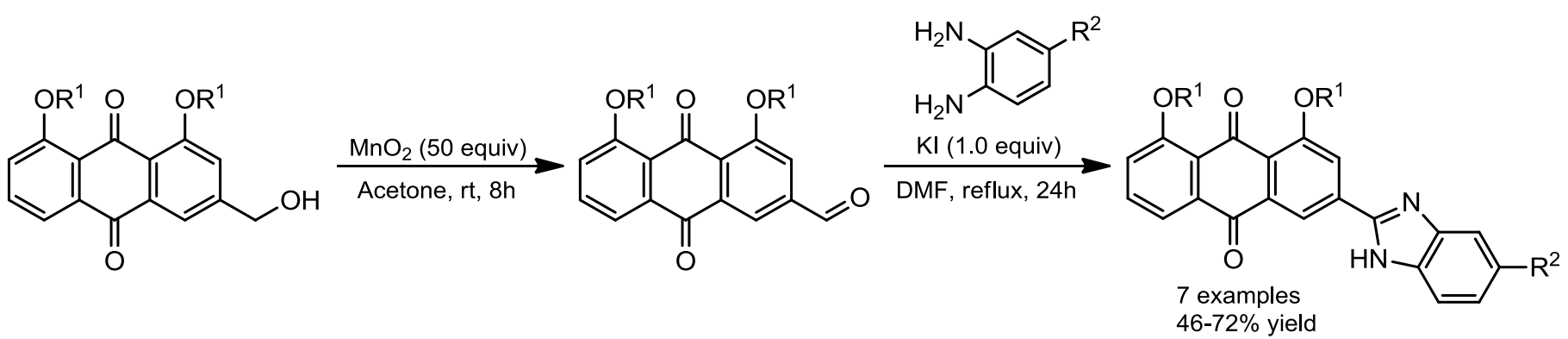

Keywords: Aloe-emodin, benzimidazole, oxidative cyclization, antimicrobial, cytotoxicity 


\section{Introduction}

The use of natural products as starting points for developing novel hit compounds is continuing to be a valuable strategy in the search for new bioactive compounds. ${ }^{1-3}$ The anthraquinone moiety occurs in many biological active molecules with antiviral, antifungal, antibacterial and antiparasitic properties, such as chrysophanol 2 (Fig 1)..$^{4-6}$ One of these molecules is aloe-emodin 1 (3-hydroxymethyl-1,8-dihydroxy-9,10antraquinone) (Figure 1), which is a well-known compound isolated from Aloe vera leaves and widely used as an intermediate in the preparation of therapeutically active compounds such as rhein $\mathbf{2}$ and diacerein $\mathbf{3}$, and anthracycline type antibiotics. ${ }^{7,8}$ Aloe-emodin and derivatives thereof have been described to be useful in the treatment of cancer and psoriasis, and as antifungal, antiviral and antiplasmodial agents. ${ }^{5,9,10}$

Although several transformations of aloe-emodin have been described, ${ }^{11,10,12-16}$ the transformation of the hydroxymethyl group to a heterocyclic moiety has received very little attention. ${ }^{17,18}$ Therefore, attempts were performed to synthesize benzimidazole derivatives of aloe-emodin to boost or tune its biological properties. Benzimidazoles occur as core structure in many pharmaceutical compounds with a diversity of bioactivities, including antiviral, antibacterial, antitumor, anti-Alzheimer, and anti-inflammatory activities. ${ }^{19-22}$<smiles>O=C1c2cc(CO)cc(O)c2C(=O)c2cccc(O)c21</smiles>

Aloe-emodin 1<smiles>O=C(O)c1cc(O)c2c(c1)C(=O)c1cccc(O)c1C2=O</smiles>

Rhein 3<smiles>Cc1cc(O)c2c(c1)C(=O)c1cccc(O)c1C2=O</smiles>

Chrysophanol 2<smiles>CC(=O)Oc1cccc2c1C(=O)c1c(OC(C)=O)cc(C(=O)O)cc1C2=O</smiles>

Diacerein 4

Figure 1. Structures of Aloe-emodin, chrysophanol, rhein and diacerein.

One of the general methods for the synthesis of benzimidazoles is the coupling of $o$-aryldiamines with carboxylic acids or their derivatives under dehydrating conditions. ${ }^{23}$ Also the reaction of o-phenylenediamines with aldehydes in the presence of oxidants and catalysts has been described. Herein, we report the synthesis of benzimidazoles derived from aloe-emodin carbaldehyde and o-phenylenediamines via aerobic oxidation in the presence of potassium iodide as auxiliary reagent, following the conditions described by Chen et al. ${ }^{24}$

\section{Results and Discussion}

Aloe-emodin 1 was first methylated at the phenolic hydroxyl groups using dimethyl sulfate as reported in literature to afford compound $\mathbf{5}$ (Scheme 1). It was observed that an excess of dimethyl sulfate is required to allow an efficient dimethylation because of the low solubility of aloe-emodin in most organic solvents. In our hands optimal conditions used 9.8 equiv of $\mathrm{Me}_{2} \mathrm{SO}_{4}$ (added in two portions) at $0.04 \mathrm{M}$ concentration of aloe- 
emodin in acetone. Stirring the mixture longer than $24 \mathrm{~h}$ in attempts to increase the yield resulted in significant amounts of the over-methylated compound $\mathbf{6}$ as side-product. Fortunately, anthraquinone $\mathbf{6}$ is much more soluble in acetone than the desired product, which can be purified by recrystallization from cold acetone.<smiles>COCc1cc(OC)c2c(c1)C(=O)c1cccc(OC)c1C2=O</smiles>

The synthesis of the required aldehydes $\mathbf{7 a}$ and $\mathbf{7 b}$ has been reported in literature using different oxidants, including chromium reagents like pyridinium chlorochromate (PCC), TEMPO/trichloroisocyanuric acid, $\mathrm{VO}(\mathrm{acac})_{2} / \mathrm{DABCO} / \mathrm{O}_{2}$ and others. ${ }^{25,15,26,12}$ Yang-Ming et al. reported the oxidation of aloe-emodin 1 to aldehyde $7 \mathrm{a}$ in the presence of manganese dioxide in refluxing acetone. ${ }^{27}$ We synthesized aldehydes $7 \mathrm{a}$ and $\mathbf{7 b}$ by oxidation of aloe-emodin $\mathbf{1}$ or its dimethyl-protected analogue $\mathbf{5}$ with an excess of manganese dioxide in ethyl acetate (See Scheme 1) at room temperature, which resulted in the desired aldehyde 7a (gram scale reaction) within $8 \mathrm{~h}$ in high yield (91\%). The same procedure was followed using dimethylated compound 5 to give $\mathbf{7 b}$ but in lower yield.

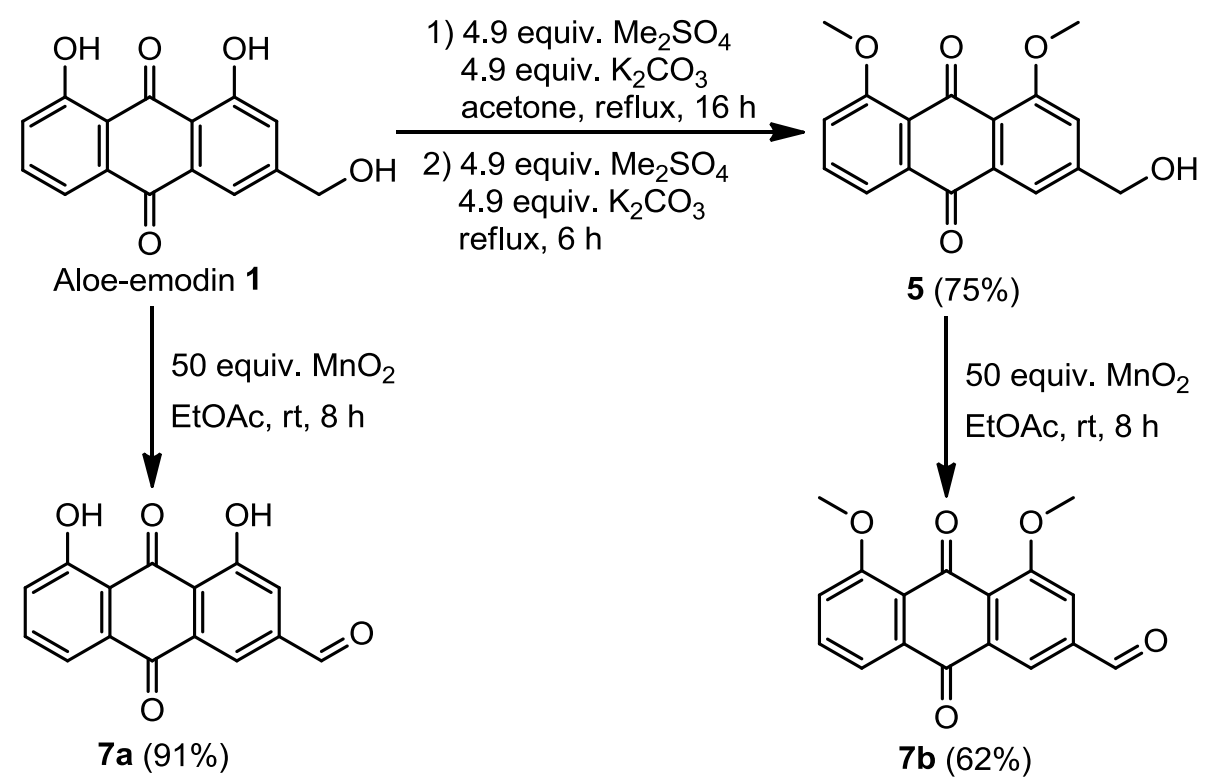

Scheme 1. Oxidation of compounds $\mathbf{1}$ and $\mathbf{5}$ to afford compounds $7 \mathbf{a}-\mathbf{b}$.

Having in hand the aldehydes $\mathbf{7 a , b}$, the corresponding benzimidazoles were prepared by reaction with aromatic diamines using potassium iodide as auxiliary reagent. A mechanism of this reaction was proposed by Chen et al. ${ }^{24}$ According to these authors, KI promotes the cyclization of $\mathrm{N}$-(o-aminoaryl)imines by attacking the $\mathrm{C}=\mathrm{N}$ bond giving a tetrahedral intermediate which can undergo 5-exo-tet cyclization instead of a direct but less plausible a 5-endo-trig cyclization on the parent imine (Scheme 2). 


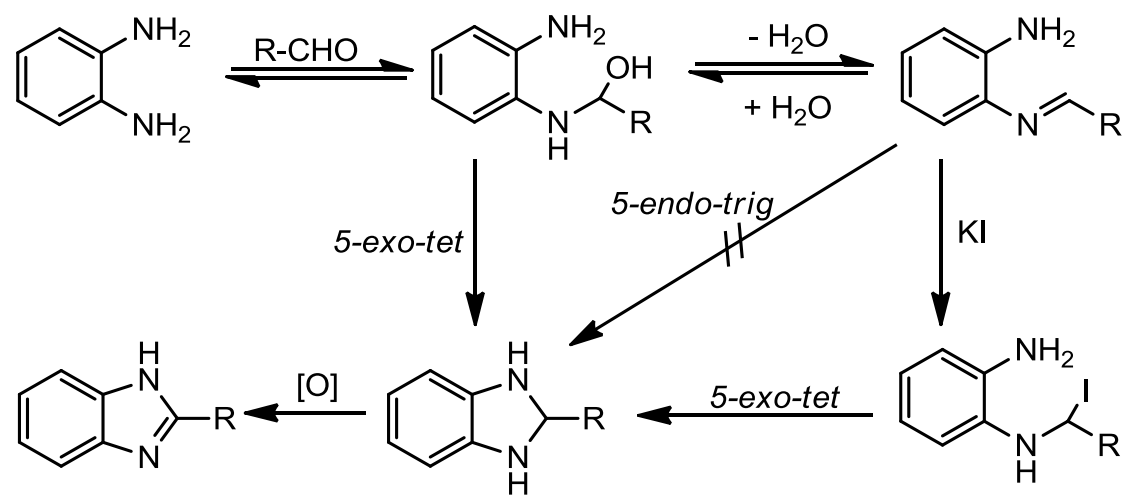

Scheme 2. Mechanism proposed by Chen et al. ${ }^{24}$

A mixture of the corresponding aldehyde and diamine was heated at $80{ }^{\circ} \mathrm{C}$ in DMF in the presence of 1 equiv of $\mathrm{KI}$ for 24 hours. Although $\mathrm{KI}$ is regenerated and could act as catalyst, it was found that raising the amount to 1 equiv gave superior results. The use of catalytic amounts of $\mathrm{KI}(10 \mathrm{~mol} \%)$ resulted in a longer reaction time and poor yields. The reaction using aldehyde 7 a resulted in the corresponding benzimidazolyl derivatives $\mathbf{8 a - c}$ in $\mathbf{4 6 - 6 6 \%}$ yield, while using aldehyde $\mathbf{7 b}$ gave compounds $\mathbf{8 d - g}$ in $\mathbf{5 0 - 7 2 \%}$ yield (See Table 1 ). All compounds were purified by recrystallization or column chromatography, depending on their solubility. Compounds $\mathbf{8 a}$ and $\mathbf{8 b}$ have a poor solubility in common organic solvents such as chloroform and acetone, while compounds $\mathbf{8} \mathbf{d}$ and $\mathbf{8 f}$ were more soluble.

Table 1. Oxidative cyclization of aldehydes 7a-b with aromatic diamines affording benzimidazoles 8a-g

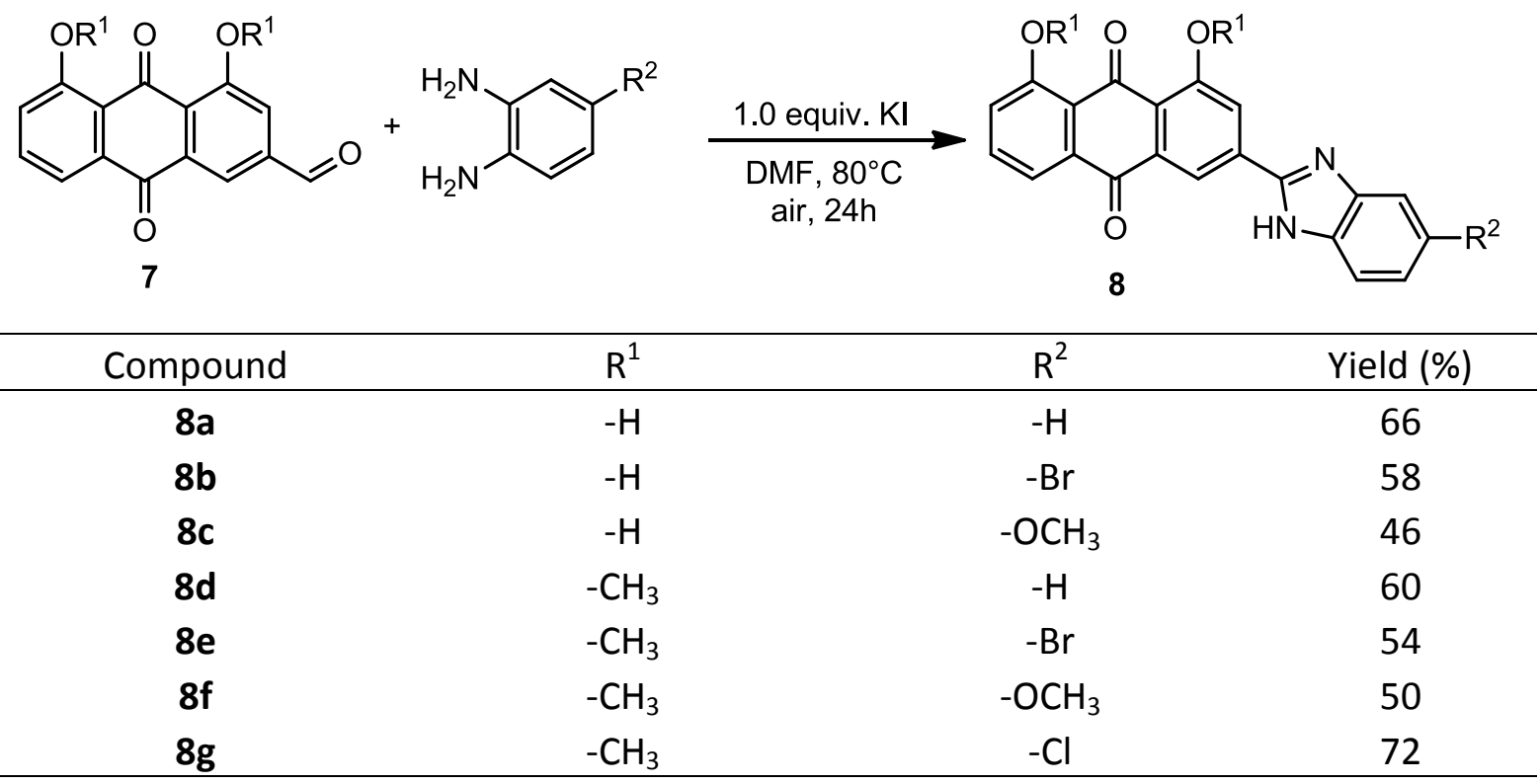

The synthesis of benzimidazole $\mathbf{8 d}$ was previously reported using sodium metabisulfite. ${ }^{17}$ The reaction was conducted at $120^{\circ} \mathrm{C}$ for 2 hours, no yield was reported. Compound 8 a was obtained by demethylation of compound $\mathbf{8 d}$ with concentrated hydrobromic acid in acetic acid. Only the melting point of both compounds was reported. 
Table 2. Activities of synthesized compounds against S. aureus, E. coli, C. albicans, and A. fumigatus and cytotoxicity against Human Lung Fibroblasts (MRC-5)

\begin{tabular}{llllll}
\hline \multirow{2}{*}{ Comp } & $\mathrm{IC}_{50}(\mu \mathrm{M})$ & & & & \\
\cline { 2 - 6 } & S. aureus & E. coli & C. albicans & A. fumigatus & MRC-5 \\
\hline std & $0.04^{\mathrm{a}}$ & $0.58^{\mathrm{a}}$ & $0.74^{\mathrm{b}}$ & $0.31^{\mathrm{c}}$ & $8.32^{\mathrm{d}}$ \\
$\mathbf{5}$ & $>128.00$ & $>128.00$ & $>128.00$ & $>128.00$ & 25.40 \\
$\mathbf{7 a}$ & 2.06 & $>128.00$ & $>128.00$ & $>128.00$ & 11.96 \\
$\mathbf{7 b}$ & $>128.00$ & $>128.00$ & $>128.00$ & $>128.00$ & 36.76 \\
$\mathbf{8 a}$ & 64.91 & $>128.00$ & $>128.00$ & $>128.00$ & 41.78 \\
$\mathbf{8 b}$ & 33.37 & $>128.00$ & $>128.00$ & $>128.00$ & 80.63 \\
$\mathbf{8 c}$ & $>128.00$ & $>128.00$ & $>128.00$ & $>128.00$ & $>128.00$ \\
$\mathbf{8 d}$ & $>128.00$ & $>128.00$ & $>128.00$ & $>128.00$ & 2.00 \\
$\mathbf{8 e}$ & $>128.00$ & $>128.00$ & $>128.00$ & $>128.00$ & $>128.00$ \\
$\mathbf{8 f}$ & 16.11 & $>128.00$ & $>128.00$ & $>128.00$ & $<0.50$ \\
$\mathbf{8 g}$ & $>128.00$ & $>128.00$ & $>128.00$ & $>128.00$ & 32.00 \\
\hline
\end{tabular}

${ }^{a}$ Doxycycline. ${ }^{b}$ Flucytosine. ${ }^{c}$ Terbinafine. ${ }^{d}$ Tamoxifen.

The antimicrobial activity and cytotoxicity of the synthesized compounds were tested in vitro. Results of antibacterial and antifungal activities are shown in Table 2.

Table 3. Activities of synthesized compounds against T. cruzi, T. b. brucei, T. brucei rhodesiense, and $L$. infantum

\begin{tabular}{lllll}
\hline \multirow{2}{*}{ Comp. } & $\mathrm{IC}_{50}(\mu \mathrm{M})$ & & & \\
\cline { 2 - 5 } & L. infantum & T. cruzi & T. b. bruc & T. b. rhodes \\
\hline std & $10.77^{\mathrm{a}}$ & $1.78^{\mathrm{b}}$ & $0.02^{\mathrm{c}}$ & $0.03^{\mathrm{c}}$ \\
$\mathbf{5}$ & 48.11 & $>128.00$ & $>128.00$ & 68.32 \\
$\mathbf{7 a}$ & 4.06 & 4.36 & 4.12 & 4.03 \\
$\mathbf{7 b}$ & 54.17 & $>128.00$ & 63.50 & 75.30 \\
$\mathbf{8 a}$ & 4.06 & $>128.00$ & 46.25 & 29.58 \\
$\mathbf{8 b}$ & 19.03 & 54.26 & 64.00 & 22.17 \\
$\mathbf{8 c}$ & 64.91 & $>128.00$ & $>128.00$ & 68.90 \\
$\mathbf{8 d}$ & 25.40 & 3.60 & 64.91 & 4.90 \\
$\mathbf{8 e}$ & $>128.00$ & $>128.00$ & $>128.00$ & 96.08 \\
$\mathbf{8 f}$ & 3.41 & $<0.50$ & 0.90 & 0.98 \\
$\mathbf{8 g}$ & 21.53 & 48.11 & $>128.00$ & 12.03 \\
\hline
\end{tabular}

${ }^{a}$ Miltefosine. ${ }^{b}$ Benznidazol. ${ }^{c}$ Suramine.

Cytotoxicity against MRC-5 cells (human fetal lung fibroblasts) was evaluated as well as to assess selectivity. Antibacterial activity was tested against both Gram-positive and Gram-negative bacteria, $S$. aureus and $E$. coli respectively. Compound $7 \mathrm{a}\left(\mathrm{IC}_{50}=2.06 \mu \mathrm{M}\right)$ was the most active against $S$. aureus, followed by compounds 8 f $\left(I C_{50}=16.11 \mu \mathrm{M}\right)$ and $8 \mathbf{b}\left(I C_{50}=33.37 \mu \mathrm{M}\right)$ but still much less active than the standard doxycycline. 
Antiprotozoal activity evaluation of the synthesized compounds against Leishmania infantum, Trypanosoma cruzi, Trypanosoma brucei and Trypanosoma brucei rhodesiense was also conducted. In general, compounds with the highest antiprotozoal activity $(\mathbf{7 a}, \mathbf{8 f}$ and $\mathbf{8 d})$ also showed the highest cytotoxicity on MRC-5 cells, revealing this activity to be quite aspecific. Compound 8 a showed good activity $\left(\mathrm{IC}_{50}=4.06 \mu \mathrm{M}\right)$ against $L$. infantum, with a Selectivity Index (ratio of the cytotoxicity on MRC-5 $\left(\mathrm{IC}_{50}\right)$ to the antimicrobial activity $\left.\left(\mathrm{IC}_{50}\right)\right)$ of 10.29 .

\section{Conclusions}

Benzimidazolic derivatives of aloe-emodin were obtained in good to moderate yields using a simple procedure. In vitro antimicrobial activity of the synthesized compounds was tested. The compounds showed no activity against E. coli, C. albicans or A. fumigatus. Compounds $7 \mathbf{a}$ and $\mathbf{8 f}$ were the most active against $S$. aureus but showed high cytotoxicity on MRC- 5 cells. Most of the compounds showed either low to moderate antiprotozoal activity, although it was quite aspecific. Compound 8a showed the highest specificity against Leishmania infantum with $\mathrm{IC}_{50}=4.06 \mu \mathrm{M}$ and selectivity index of 10.29.

\section{Experimental Section}

General. Column chromatography purifications were conducted on silica gel 60 (40-63 $\mu \mathrm{m}$; Grace Davisil). TLC was carried out on plastic TLC sheets, precoated with silica gel 60F254 (Merck); the spots were visualized under UV light $(\lambda=254 \mathrm{~nm})$. Melting points were acquired on a Buchi Melting Point B-540. IR absorption spectra were recorded on a Thermo Nicolet Avatar 370 FT-IR spectrophotometer. ${ }^{1} \mathrm{H}$ NMR and ${ }^{13} \mathrm{C}$ NMR spectra were recorded on a Bruker Avance DRX 250 console and a Bruker Avance II 500 console at 250 or 63 $\mathrm{MHz}$ and 500 or $125 \mathrm{MHz}$, respectively. The deuterated solvent is mentioned in the analysis section and tetramethylsilane was used as an internal standard. Chemical shifts ( $\delta$ ) are given in parts per million (ppm). High Resolution Mass Spectrometry was conducted on a Waters Micromass QTof in ES+ mode, using reserpine as a reference. All reagents were purchased and used without further purifications. Unless explicitly mentioned, reactions were performed without specific drying of solvents or use of an inert atmosphere. Ethyl acetate for chromatography purposes was distilled prior to use.

Synthesis of 3-(hydroxymethyl)-1,8-dimethoxyanthracene-9,10-dione (5). This product was synthesized similarly to a literature procedure. ${ }^{11} \mathrm{~A}$ solution of aloe-emodin $(1 \mathrm{~g}, 3.7 \mathrm{mmol})$ in acetone $(200 \mathrm{~mL})$ was added with $\mathrm{K}_{2} \mathrm{CO}_{3}(2.5 \mathrm{~g}, 18.1 \mathrm{mmol})$ and dimethyl sulfate $(1.73 \mathrm{~mL}, 18.2 \mathrm{mmol})$, and then refluxed overnight. The reaction mixture was added then with more $\mathrm{K}_{2} \mathrm{CO}_{3}(2.5 \mathrm{~g})$ and dimethyl sulfate $(1.73 \mathrm{~mL})$ and refluxed for 6 hours. The reaction was monitored by TLC. After the completion of reaction, the yielded mixture was cooled to room temperature and filtered. The filtrate was dried under vacuum to afford a yellow solid. Recrystallization of the yellow solid from acetone gave compound $\mathbf{2}$ as yellow needles, yield 75\%; m.p. 224 $225{ }^{\circ} \mathrm{C} ;{ }^{1} \mathrm{H}$ NMR $\left(250 \mathrm{MHz}, d_{6}\right.$-DMSO) $\delta(\mathrm{ppm}): 3.91\left(\mathrm{~s}, 6 \mathrm{H}, 2 \times \mathrm{OCH}_{3}\right), 4.62\left(\mathrm{~s}, 2 \mathrm{H}, \mathrm{CH}_{2} \mathrm{O}\right), 5.53(1 \mathrm{H}, \mathrm{OH}), 7.21$ $(1 \mathrm{H}, \mathrm{s}, \mathrm{CH}$ aromatic), $7.30(1 \mathrm{H}, \mathrm{d}, \mathrm{CH}$ aromatic), $7.58(1 \mathrm{H}, \mathrm{s}, \mathrm{CH}$ aromatic), $7.61(1 \mathrm{H}, \mathrm{d}, \mathrm{CH}$ aromatic), $7.74(1 \mathrm{H}$, $\mathrm{dd}, \mathrm{CH}$ aromatic). The obtained spectrum is consistent with literature data. ${ }^{12}$ 
General procedure for the synthesis of the aldehydes 7a-b. To a mixture of aloe-emodin ( $1 \mathrm{~g}, 3.7 \mathrm{mmol}$ ) (or compound 2) and manganese dioxide (16.09 g, $185 \mathrm{mmol}$ ) was added $1000 \mathrm{~mL}$ of ethyl acetate. The reaction mixture was stirred for 8 hours, filtered, and the solvent was evaporated in vacuo. The product was purified by recrystallization from acetone.

4,5-Dihydroxy-9,10-dioxo-9,10-dihydroanthracene-2-carbaldehyde (7a). Reddish solid, M.p.:208-209 ${ }^{\circ} \mathrm{C}$ (lit. 208-210 ${ }^{\circ} \mathrm{C}$ ), yield: 91.1\%; ${ }^{1} \mathrm{H}$ NMR: $\left(250 \mathrm{MHz}, \mathrm{DMSO}-d_{6}\right) \delta(\mathrm{ppm}): 11.93(\mathrm{~s}, 1 \mathrm{H}, \mathrm{OH}), 11.89(\mathrm{~s}, 1 \mathrm{H}, \mathrm{OH}), 10.11(\mathrm{~s}$, $1 \mathrm{H},-\mathrm{CHO}), 8.11$ (d, $1 \mathrm{H}, \mathrm{CH}$ aromatic), 7.86-7.72 (m, 3H, $\mathrm{CH}$ aromatic), 7.41 (d, $1 \mathrm{H}, \mathrm{CH}$ aromatic). The obtained spectrum is consistent with literature data. ${ }^{12}$

4,5-Dimethoxy-9,10-dioxo-9,10-dihydroanthracene-2-carbaldehyde (7b). Yellow solid, yield 62.1\%; m.p. 195$196{ }^{\circ} \mathrm{C}$ (lit. 198-200 ${ }^{\circ} \mathrm{C}$ ); ${ }^{1} \mathrm{H}$ NMR (250 MHz, DMSO- $\left.d_{6}\right) \delta(\mathrm{ppm}): 3.92$ (s, 3H, OCH $), 4.00\left(\mathrm{~s}, 3 \mathrm{H}, \mathrm{OCH}_{3}\right), 7.56(\mathrm{~d}$, $1 \mathrm{H}, \mathrm{CH}$ aromatic), 7.75 (m, 2H, $2 \times \mathrm{CH}$ aromatic), 7.92 (s, 1H, $\mathrm{CH}$ aromatic), 8.18 (d, 1H, CH aromatic), 10.15 (s, $1 \mathrm{H}, \mathrm{CHO})$. The obtained spectrum is consistent with literature data. ${ }^{15}$

General procedure for the synthesis of the benzimidazoles 8a-g. An ortho-phenylenediamine derivative (1.0 mmol; 1.0 equiv) and an aldehyde 7 (1.0 mmol; 1.0 equiv) were dissolved in DMF (20 mL). Potassium iodide (1.0 mmol; 1.0 equiv) was added. The resulting reaction mixture was stirred at $80^{\circ} \mathrm{C}$ in an open flask, and the reaction progress was monitored by TLC. On the completion of the reaction, the mixture was cooled to room temperature, poured into water $(150 \mathrm{~mL})$ and extracted with ethyl acetate $(3 \times 75 \mathrm{~mL})$. The crude product obtained was purified by column chromatography on silica gel to afford the corresponding benzimidazole 8; except compounds $\mathbf{8 a - c}$, which were purified by recrystallization due to their low solubility in common organic solvents.

3-(1H-Benzo[d]imidazol-2-yl)-1,8-dihydroxyanthracene-9,10-dione (8a). Red solid, yield 66\%; M.p. >350 ${ }^{\circ} \mathrm{C}$; IR (cm ${ }^{-1}$, neat): $3300,1621,1572,1454,1266,1207,1152,1088,1020,814,740,724,666 ;{ }^{1} \mathrm{H} \mathrm{NMR}(500 \mathrm{MHz}$, DMSO- $\left.d_{6}\right) \delta(\mathrm{ppm}): 13.33(\mathrm{~s}, 1 \mathrm{H}, \mathrm{NH}), 11.93(\mathrm{~s}, 2 \mathrm{H}, \mathrm{OH}), 8.51(\mathrm{~s}, 1 \mathrm{H}, \mathrm{CH}$ aromatic), 8.06 (s, $1 \mathrm{H}, \mathrm{CH}$ aromatic), $7.74\left(\mathrm{~m}, 5 \mathrm{H}, \mathrm{CH}\right.$ aromatic), 7.39 (s, $1 \mathrm{H}, \mathrm{CH}$ aromatic), 7.26 (s, 1H, CH aromatic). $\left.{ }^{13} \mathrm{C} \mathrm{NMR} \mathrm{(125} \mathrm{MHz,} \mathrm{DMSO-} d_{6}\right)$ $\delta$ (ppm): $191.93(\mathrm{C}=\mathrm{O}), 181.90(\mathrm{C}=\mathrm{O}), 162.42(\mathrm{C}-\mathrm{OH}), 162.09(\mathrm{C}-\mathrm{OH}), 149.34(\mathrm{C}=\mathrm{N}), 138.59$ (C aromatic), 138.28 (C aromatic), 138.10 (C aromatic), 134.82 (C aromatic), 133.97 (C aromatic), 125.23 ( $2 \times \mathrm{C}$ aromatic), 121.06 ( $2 \times \mathrm{C}$ aromatic), 120.14 ( $3 \times \mathrm{C}$ aromatic), 117.65 ( $\times$ C aromatic), 117.06 (C aromatic), 116.76 (C aromatic). HRMS $\left[\mathrm{C}_{21} \mathrm{H}_{12} \mathrm{~N}_{2} \mathrm{O}_{4}+\mathrm{H}\right]^{+}$calculated 357.0876 found 357.0859

3-(5-Bromo-1H-benzo[d]imidazol-2-yl)-1,8-dihydroxyanthracene-9,10-dione (8b). Brown-yellow solid, yield 58\%; m.p. 383-384 ${ }^{\circ} \mathrm{C}$; IR (cm ${ }^{-1}$, neat): 3300, 3072, 1623, 1455, 1384, 1263, 1206, 1156, 1013, 817, 759, 722, 667; ${ }^{1} \mathrm{H}$ NMR (500 MHz, DMSO- $\left.d_{6}\right) \delta(\mathrm{ppm}): 12.44(\mathrm{~s}, 2 \mathrm{H}, \mathrm{OH}), 8.41(\mathrm{~s}, 1 \mathrm{H}, \mathrm{CH}$ aromatic), $8.00(\mathrm{~s}, 1 \mathrm{H}, \mathrm{CH}$ aromatic), $7.88-7.65$ (m, 3H, CH aromatic), 7.55 (d, 1H, CH aromatic), 7.35 (t, 2H, CH aromatic). ${ }^{13} \mathrm{C}$ NMR (125 $\mathrm{MHz}$, DMSO- $\left.d_{6}\right) \delta(\mathrm{ppm}): 191.27(\mathrm{C}=\mathrm{O}), 181.14(\mathrm{C}=\mathrm{O}), 161.59(\mathrm{C}-\mathrm{OH}), 161.39(\mathrm{C}-\mathrm{OH}), 149.81(\mathrm{C}=\mathrm{N}), 137.47$ ( $2 \times \mathrm{C}$ aromatic), 137.03 (C aromatic), 134.16 (C aromatic), 133.25 (C aromatic), 124.57 ( $2 \times \mathrm{C}$ aromatic), 120.58 ( $2 \times \mathrm{C}$ aromatic), 119.50 ( $2 \times \mathrm{C}$ aromatic), 117.02 ( $2 \times \mathrm{C}$ aromatic), 116.64 (C aromatic), 116.07 ( $2 \times \mathrm{C}$ aromatic). $\mathrm{HRMS}\left[\mathrm{C}_{21} \mathrm{H}_{11} \mathrm{BrN}_{2} \mathrm{O}_{4}+\mathrm{H}\right]^{+}$calculated 434.9981 found 434.9962

1,8-Dihydroxy-3-(5-methoxy-1H-benzo[d]imidazol-2-yl)anthracene-9,10-dione (8c). Black solid, yield 46\%; m.p. $328-329^{\circ} \mathrm{C}$; IR (cm ${ }^{-1}$, neat): 3321, 1620, 1454, 1385, 1284, 1211, 1158, 1091, 829, 760, 724, 666; ${ }^{1} \mathrm{H}$ NMR $\left(500 \mathrm{MHz}, \mathrm{DMSO}-d_{6}\right) \delta(\mathrm{ppm}): 13.03(\mathrm{~s}, 1 \mathrm{H}, \mathrm{NH}), 11.88(\mathrm{~s}, 2 \mathrm{H}, \mathrm{OH}), 8.29(\mathrm{~s}, 1 \mathrm{H}, \mathrm{CH}$ aromatic), $7.86(\mathrm{~s}, 1 \mathrm{H}, \mathrm{CH}$ aromatic), $7.70(\mathrm{~d}, 2 \mathrm{H}, \mathrm{CH}$ aromatic), $7.36(\mathrm{~d}+\mathrm{s}, 2 \mathrm{H}, \mathrm{CH}$ aromatic), $6.84(\mathrm{~d}+\mathrm{s}, 2 \mathrm{H}, \mathrm{CH}$ aromatic), $3.75(\mathrm{~s}, 3 \mathrm{H}$, $\left.\mathrm{OCH}_{3}\right) \cdot{ }^{13} \mathrm{C}$ NMR $\left(125 \mathrm{MHz}, \mathrm{DMSO}-d_{6}\right) \delta(\mathrm{ppm}): 191.85(\mathrm{C}=\mathrm{O}), 181.80(\mathrm{C}=\mathrm{O}), 162.34(\mathrm{C}-\mathrm{OH}), 162.03(\mathrm{C}-\mathrm{OH})$, $157.28\left(\mathrm{C}-\mathrm{OCH}_{3}\right), 148.65(\mathrm{C}=\mathrm{N}), 138.30$ (C aromatic), 138.02 (2×C aromatic), 134.65 (C aromatic), 133.91 (C 
aromatic), 125.14 ( $2 \times \mathrm{C}$ aromatic), $120.38(2 \times \mathrm{C}$ aromatic), $120.11(2 \times \mathrm{C}$ aromatic), $117.43(2 \times \mathrm{C}$ aromatic), 116.63 (C aromatic), 113.89 (C aromatic), $56.08\left(\mathrm{OCH}_{3}\right)$. HRMS $\left[\mathrm{C}_{22} \mathrm{H}_{14} \mathrm{~N}_{2} \mathrm{O}_{5}+\mathrm{H}\right]^{+}$calculated 387.0982 found 387.0969

3-(1H-Benzo[d]imidazol-2-yl)-1,8-dimethoxyanthracene-9,10-dione (8d). Brown solid, yield 60\%; m.p. 190$191^{\circ} \mathrm{C}$; IR (cm ${ }^{-1}$, neat): 3500, 3090, 2922, 1650, 1603, 1583, 1459, 1328, 1281, 1231, 1070, 1001, 910, 861, 792, 744, 716; ${ }^{1} \mathrm{H}$ NMR (500 MHz, DMSO- $\left.d_{6}\right) \delta(\mathrm{ppm}): 13.37(\mathrm{~s}, 1 \mathrm{H}, \mathrm{NH}), 8.52(\mathrm{~s}, 1 \mathrm{H}, \mathrm{CH}$ aromatic), $8.26(\mathrm{~s}, 1 \mathrm{H}, \mathrm{CH}$ aromatic), $7.78(\mathrm{~m}, 3 \mathrm{H}, \mathrm{CH}$ aromatic), $7.61(\mathrm{~d}, 1 \mathrm{H}, \mathrm{CH}$ aromatic), $7.58(\mathrm{~d}, 1 \mathrm{H}, \mathrm{CH}$ aromatic), $7.30(\mathrm{t}, 1 \mathrm{H}, \mathrm{CH}$ aromatic), $7.26\left(\mathrm{t}, 1 \mathrm{H}, \mathrm{CH}\right.$ aromatic), $4.07\left(\mathrm{~s}, 3 \mathrm{H}, \mathrm{OCH}_{3}\right), 3.94\left(\mathrm{~s}, 3 \mathrm{H}, \mathrm{OCH}_{3}\right) .{ }^{13} \mathrm{C} \mathrm{NMR}\left(125 \mathrm{MHz}, \mathrm{DMSO}-d_{6}\right) \delta$ (ppm): $183.74(\mathrm{C}=\mathrm{O}), 181.45(\mathrm{C}=\mathrm{O}), 159.93\left(\mathrm{C}-\mathrm{OCH}_{3}\right), 159.47\left(\mathrm{C}-\mathrm{OCH}_{3}\right), 150.02(\mathrm{C}=\mathrm{N}), 144.37(\mathrm{C} 3), 135.84(\mathrm{C}$ aromatic), 135.63 (C aromatic), 135.53 (C aromatic), 135.03 (C aromatic), 134.75 (C aromatic), 124.54 (C aromatic), 124.17 (C aromatic), 124.14 (C aromatic), 122.93 (C aromatic), 120.04 (C aromatic), 119.78 (C aromatic), 118.95 (C aromatic), 116.55 (C aromatic), 116.11 (C aromatic), 112.43 (C aromatic), $57.26\left(\mathrm{OCH}_{3}\right)$, $57.03\left(\mathrm{OCH}_{3}\right)$. HRMS $\left[\mathrm{C}_{23} \mathrm{H}_{16} \mathrm{~N}_{2} \mathrm{O}_{4}+\mathrm{H}\right]^{+}$calculated 385.1188 found 385.1183

3-(5-Bromo-1H-benzo[d]imidazol-2-yl)-1,8-dimethoxyanthracene-9,10-dione (8e). Brown-yellow solid, yield 54\%; m.p. 275-276 ${ }^{\circ} \mathrm{C}$; IR (cm ${ }^{-1}$, neat): 3090, 1655, 1604, 1584, 1445, 1322, 1274, 1239, 1066, 1011, 977, 916, 799, 755, 724; ${ }^{1} \mathrm{H}$ NMR (500 MHz, DMSO- $\left.d_{6}\right) \delta$ (ppm): $13.39(\mathrm{~s}, 1 \mathrm{H}, \mathrm{NH}), 8.36(\mathrm{~s}, 1 \mathrm{H}, \mathrm{CH}$ aromatic), $8.09(\mathrm{~s}, 1 \mathrm{H}$, $\mathrm{CH}$ aromatic), 7.76 (s, 1H, CH aromatic), $7.72-7.63(\mathrm{~m}, 2 \mathrm{H}, \mathrm{CH}$ aromatic), 7.54 (d, 1H, CH aromatic), 7.48 (d, $1 \mathrm{H}, \mathrm{CH}$ aromatic), 7.32 (d, $1 \mathrm{H}, \mathrm{CH}$ aromatic), $4.01\left(\mathrm{~s}, 3 \mathrm{H}, \mathrm{OCH}_{3}\right), 3.90\left(\mathrm{~s}, 3 \mathrm{H}, \mathrm{OCH}_{3}\right) .{ }^{13} \mathrm{C} \mathrm{NMR}(125 \mathrm{MHz}, \mathrm{DMSO}-$ $\left.d_{6}\right) \delta(\mathrm{ppm}): 183.43(\mathrm{C}=\mathrm{O}), 181.22(\mathrm{C}=\mathrm{O}), 159.78\left(\mathrm{C}-\mathrm{OCH}_{3}\right), 159.41\left(\mathrm{C}-\mathrm{OCH}_{3}\right), 151.09(\mathrm{C}=\mathrm{N}), 135.28(\mathrm{C}$ aromatic), 134.91 (C aromatic), 134.85 (C aromatic), 134.56 (C aromatic), 126.25 (C aromatic), 124.51 (C aromatic), 123.94 (C aromatic), 119.58 ( $2 \times \mathrm{C}$ aromatic), 118.85 ( $2 \times \mathrm{C}$ aromatic), 116.57 ( $2 \times \mathrm{C}$ aromatic), $116.03(2 \times \mathrm{C}$ aromatic), 115.59 (C aromatic), $57.15\left(\mathrm{OCH}_{3}\right), 56.93\left(\mathrm{OCH}_{3}\right)$. HRMS $\left[\mathrm{C}_{23} \mathrm{H}_{15} \mathrm{BrN}_{2} \mathrm{O}_{4}+\mathrm{H}\right]^{+}$calculated 463.0293 found 463.0288

1,8-Dimethoxy-3-(5-methoxy-1H-benzo[d]imidazol-2-yl)anthracene-9,10-dione (8f). Black solid, yield 50\%; m.p. $190-191^{\circ} \mathrm{C}$; IR (cm ${ }^{-1}$, neat): 3152, 1662, 1585, 1445, 1304, 1277, 1234, 1159, 1065, 1011, 977, 912, 814, 752, 714; ${ }^{1} \mathrm{H}$ NMR (500 MHz, DMSO- $\left.d_{6}\right) \delta(\mathrm{ppm}): 13.21(\mathrm{~s}, 1 \mathrm{H}, \mathrm{NH}), 8.45(\mathrm{~s}, 1 \mathrm{H}, \mathrm{CH}$ aromatic), $8.18(\mathrm{~s}, 1 \mathrm{H}, \mathrm{CH}$ aromatic), $7.82-7.71(\mathrm{~m}, 3 \mathrm{H}, \mathrm{CH}$ aromatic), $7.63(\mathrm{~d}, 1 \mathrm{H}, \mathrm{CH}$ aromatic), 7.56 (d, 1H, CH aromatic), $7.48(\mathrm{~d}, 1 \mathrm{H}$, $\mathrm{CH}$ aromatic), $4.05(\mathrm{~s}, 3 \mathrm{H}), 3.93(\mathrm{~s}, 3 \mathrm{H}), 3.84(\mathrm{~s}, 3 \mathrm{H}) .{ }^{13} \mathrm{C} N M R\left(125 \mathrm{MHz}, \mathrm{DMSO}-d_{6}\right) \delta(\mathrm{ppm}): 183.11(\mathrm{C}=\mathrm{O})$, $180.76(\mathrm{C}=\mathrm{O}), 159.25\left(\mathrm{C}-\mathrm{OCH}_{3}\right), 158.78\left(\mathrm{C}-\mathrm{OCH}_{3}\right), 156.77\left(\mathrm{C}-\mathrm{OCH}_{3}\right), 148.44(\mathrm{C}=\mathrm{N}), 138.31$ ( $\mathrm{C}$ aromatic), 135.92 (C aromatic), 135.14 (C aromatic), 134.83 (C aromatic), 134.33 (C aromatic), 134.06 (C aromatic), 123.48 (C aromatic), 119.96 (C aromatic), 119.09 (C aromatic), 118.26 (C aromatic), 115.65 (C aromatic), 115.45 (C aromatic), 115.16 (C aromatic), 114.94 (C aromatic), 113.64 (C aromatic), $56.53\left(\mathrm{OCH}_{3}\right), 56.34\left(\mathrm{OCH}_{3}\right), 55.50$ $\left(\mathrm{OCH}_{3}\right)$. HRMS $\left[\mathrm{C}_{24} \mathrm{H}_{18} \mathrm{~N}_{2} \mathrm{O}_{5}+\mathrm{H}\right]^{+}$calculated 415.1294 found 415.1288

3-(5-Chloro-1H-benzo[d]imidazol-2-yl)-1,8-dimethoxyanthracene-9,10-dione (8g). Orange solid, yield 72\%; m.p. 266-267 C; IR (cm ${ }^{-1}$, neat): 3467, 1658, 1585, 1445, 1325, 1273, 1241, 1066, 1012, 977, 913, 802, 755, 725; ${ }^{1} \mathrm{H}$ NMR $\left(500 \mathrm{MHz}, \mathrm{DMSO}-d_{6}\right) \delta(\mathrm{ppm}): 8.46(\mathrm{~s}, 1 \mathrm{H}, \mathrm{CH}$ aromatic), $8.19(\mathrm{~s}, 1 \mathrm{H}, \mathrm{CH}$ aromatic), $7.76(\mathrm{t}, 1 \mathrm{H}$, $\mathrm{CH}$ aromatic), 7.72 (d, 1H, CH aromatic), 7.67 (d, 1H, CH aromatic), 7.55 (d, 1H, CH aromatic), $7.29(\mathrm{~d}, 1 \mathrm{H}, \mathrm{CH}$ aromatic), $4.05\left(\mathrm{~s}, 3 \mathrm{H}, \mathrm{OCH}_{3}\right), 3.93\left(\mathrm{~s}, 3 \mathrm{H}, \mathrm{OCH}_{3}\right) .{ }^{13} \mathrm{C} \mathrm{NMR}\left(125 \mathrm{MHz}, \mathrm{DMSO}-d_{6}\right) \delta(\mathrm{ppm}): 182.87(\mathrm{C}=\mathrm{O}), 180.66$ $(\mathrm{C}=\mathrm{O}), 159.20\left(\mathrm{C}-\mathrm{OCH}_{3}\right), 158.79\left(\mathrm{C}-\mathrm{OCH}_{3}\right), 150.50(\mathrm{C}=\mathrm{N}), 134.81$ (C aromatic), 134.36 (2×C aromatic), 133.97 (C aromatic), 133.86 (C aromatic), 127.40 (C aromatic), 124.17 (C aromatic), 123.41 (2C aromatic), 119.08 (C aromatic), 118.25 ( $2 \times \mathrm{C}$ aromatic), 116.05 (2×C aromatic), $115.60\left(2 \times \mathrm{C}\right.$ aromatic), $56.60\left(\mathrm{OCH}_{3}\right), 56.33\left(\mathrm{OCH}_{3}\right)$. HRMS $\left[\mathrm{C}_{23} \mathrm{H}_{15} \mathrm{ClN}_{2} \mathrm{O}_{4}+\mathrm{H}\right]^{+}$calculated 419.0799 found 419.0793 
Antimicrobial activity. Synthesized compounds were evaluated for antimicrobial activity in an integrated screening panel against the bacteria Staphylococcus aureus and Escherichia coli, the mold Aspergillus fumigatus, the yeast Candida albicans, and the protozoa Trypanosoma cruzi, Trypanosoma brucei, Trypanosoma brucei rhodesiense and Leishmania infantum, as previously described in Cos et al. ${ }^{4}$ The human MRC-5 cell line was also included to assess selectivity, as reported before. ${ }^{28-31}$ The $I_{50}$ values were determined from five 4-fold dilutions. The following positive controls were used: Tamoxifen (MRC-5, human fetal lung fibroblasts, ECACC 84100401) $I_{50} 8.32 \mu \mathrm{M}$; doxycycline (Staphylococcus aureus ATCC 29213) $\mathrm{IC}_{50} 0.04 \mu \mathrm{M}$; doxycycline (Escherichia coli ATCC 8739), IC $50.58 \mu \mathrm{M}$; Flucytosine (Candida albicans, B59630) $\mathrm{IC}_{50} 0.74 \mu \mathrm{M}$; terbinafin (Aspergillus fumigatus ATCC16404,) IC $\mathrm{C}_{50} 0.31 \mu \mathrm{M}$; suramine (Trypanosoma brucei, Squib 427) IC $50.02 \mu \mathrm{M}$; suramine (Trypanosoma brucei rhodesiense, STIB-900) $I_{50} 0.03 \mu \mathrm{M}$; benznidazol (Trypanosoma cruzi, Tulahuen $\mathrm{CL} 2$ ) $\mathrm{IC}_{50} 1.78 \mu \mathrm{M}$; miltefosine (Leishmania infantum, MHOM/MA/67/ITMAP263) IC $5010.77 \mu \mathrm{M}$.

Statistical analysis. IC 50 calculations were performed using regression analysis [\% inhibition vs. log (concentration)] using Sigma plot 13.0.

\section{Acknowledgements}

The Flemish Development Cooperation is acknowledged for financial support given through the Flemish Interuniversity Council-University Cooperation for Development (VLIR-UOS) in the framework of an Institutional University Cooperation programme with Universidad de Oriente, Santiago de Cuba Cuba. VUB is acknowledged for financial support (Starting Grant G.V.)

\section{Supplementary Material}

Copies of ${ }^{1} \mathrm{H}$ and ${ }^{13} \mathrm{C}$ NMR spectra of benzimidazoles $8 \mathrm{a}-\mathrm{g}$ are provided as Supplementary Material for this article.

\section{References}

1. Shen, B. Cell 2015, 163, 1297-1300.

https://doi.org/10.1016/i.cell.2015.11.031

2. Rouhi, A. M. Chem. Engin. News 2003, 81, 77-77.

https://doi.org/10.1021/cen-v081n041.p077

3. Koehn, F. E.; Carter, G. T. Discovery med. 2009, 5, 159-164.

4. Duval, J.; Pecher, V.; Poujol, M.; Lesellier, E. Industrial Crops and Products 2016, 94, 812-833. https://doi.org/10.1016/j.indcrop.2016.09.056

5. Dave, H.; Ledwani, L. Indian J Nat Prod Resour 2012, 3, 291-319.

6. Fouillaud, M.; Venkatachalam, M.; Girard-Valenciennes, E.; Caro, Y.; Dufossé, L. Mar. Drugs 2016, 14, 64. https://doi.org/10.3390/md14040064 
7. Carcasona, A.; Grimminger, W.; Hietala, P.; Zaeske, H.; Witthohn, K. US005393898A, US Patent Office, 1995

8. Mitscher, L. A. US4215062 A, US Patent Office, 1980

9. Agarwal, S. K.; Sudhir, S. S.; Sushma, V.; Sushil, K. J. Ethnopharm. 2000, 72, 43-46. https://doi.org/10.1016/S0378-8741(00)00195-1

10. Shi, D.-H.; Huang, W.; Li, C.; Wang, L.-T.; Wang, S.-F. Bioorg. Med. Chem. 2013, 21, 1064-1073. https://doi.org/10.1016/i.bmc.2013.01.015

11. Cui, X.-R.; Takahashi, K.; Shimamura, T.; Koyanagi, J.; Komada, F.; Saito, S. Chem. Pharm. Bull. 2008, 56, 497-503. https://doi.org/10.1248/cpb.56.497

12. Shi, D.-H.; Huang, W.; Li, C.; Liu, Y.-W.; Wang, S.-F. Eur. J. Med. Chem. 2014, 75, 289-296. https://doi.org/10.1016/j.ejmech.2014.01.058

13. Viayna, E.; Sola, I.; Bartolini, M.; Simone, A. D.; Tapia-Rojas, C.; Serrano, F. G.; Sabaté, R.; Juárez-Jiménez, J.; Pérez, B.; Luque, F. J.; Andrisano, V.; Clos, M. V.; Inestrosa, N. C.; Muñoz-Torrero, D. J. Med. Chem. 2014, 57, 2549-2567.

14. Yao, G.-y.; Ye, M.-y.; Huang, R.-z.; Li, Y.-j.; Pan, Y.-m.; Xu, Q.; Liao, Z.-X.; Wang, H.-s. Bioorg. Med. Chem. Lett. 2014, 24, 501-507. https://doi.org/10.1016/j.bmcl.2013.12.030

15. Liu, J.; Wu, F.; Chen, C. Bioorg. Med. Chem. Lett. 2015, 25, 5142-5146. https://doi.org/10.1016/j.bmcl.2015.10.004

16. Thimmegowda, N. R.; Park, C.; Shwetha, B.; Sakchaisri, K.; Liu, K.; Hwang, J.; Lee, S.; Jeong, S. J.; Soung, N. K.; Jang, J. H.; Ryoo, I.-J.; Ahn, J. S.; Erikson, R. L.; Kim, B. Y. Chem Biol Drug Des 2015, 85, 638-644. https://doi.org/10.1111/cbdd.12448

17. Brunavs, M.; Dell, C. P.; Dobson, D. R.; Gallagher, P. T.; Hicks, T. A.; Owton, W. M.; Smith, C. W. EP0570091A1, Eur. Pat., 1993.

18. Liang, Y.-K.; Yue, Z.-Z.; Li, J.-X.; Tan, C.; Miao, Z.-H.; Tan, W.-F.; Yang, C.-H. Eur. J. Med. Chem. 2014, 84, 505-515.

https://doi.org/10.1016/j.ejmech.2014.07.047

19. Bansal, Y.; Silakari, O. Bioorg. Med. Chem. 2012, 20, 6208-6236.

https://doi.org/10.1016/j.bmc.2012.09.013

20. Gaba, M.; Singh, S.; Mohan, C. Eur. J. Med. Chem. 2014, 76, 494-505. https://doi.org/10.1016/j.ejmech.2014.01.030

21. Singh, N.; Pandurangan, A.; Rana, K.; Anand, P.; Ahmad, A.; Tiwari, A. K. Int. Curr. Pharm. J. 2012, 1, 119127. https://doi.org/10.3329/icpj.v1i5.10284

22. Singla, P.; Luxami, V.; Paul, K. RSC Adv. 2014, 4, 12422-12440. https://doi.org/10.1039/c3ra46304d

23. Alaqeel, S. I. J Saudi Chem Soc 2017, 21, 229-237. https://doi.org/10.1016/j.jscs.2016.08.001

24. Chen, G.-F.; Shen, H.-D.; Jia, H.-M.; Zhang, L.-Y.; Kang, H.-Y.; Qi, Q.-Q.; Chen, B.-H.; Cao, J.-L.; Li, J.-T. Aust J Chem 2013, 66, 262-266. https://doi.org/10.1071/CH12458

25. Roy, B. N.; Sing, G. P.; Lathi, P. S. Indian J. Chem 2012, 51B, 988-991. 
26. Zhang, Y.; Zhang, C.; Wu, W. Dalian Gongye Daxue Xuebao 2009, 28, 13-16.

27. Yang-Ming, Z.; Diangen, Z.; Wenjie, W.; Bo, W. CN101104583B, Chinese Patent Office (SIPO), 2006

28. Cos, P.; Vlietinck, A. J.; Vanden Berghe, D.; Maes, L. J. Ethnopharmacol. 2006, 106, 290-302. https://doi.org/10.1016/j.jep.2006.04.003

29. Mesia, G. K.; Tona, G. L.; Nanga, T. H.; Cimanga, R. K.; Apers, S.; Cos, P.; Maes, L.; Pieters, L.; Vlietinck, A. J. J. Ethnopharmacol. 2008, 115, 409-415. https://doi.org/10.1016/j.jep.2007.10.028

30. Baldé, E. S.; Megalizzi, V.; Traoré, M. S.; Cos, P.; Maes, L.; Decaestecker, C.; Pieters, L.; Baldé, A. M. J. Ethnopharmacol. 2010, 130, 529-535. https://doi.org/10.1016/j.jep.2010.05.042

31. Amin, A.; Tuenter, E.; Exarchou, V.; Upadhyay, A.; Cos, P.; Maes, L.; Apers, S.; Pieters, L. Phytother. Res. 2016, 30, 1624-1633.

https://doi.org/10.1002/ptr.5663 